\title{
تأثير استراتيجية توجيه التفكير خلال القراءة (DRTA) \\ في تنمية الفهم القرائي لدى طلاب المدرسة العالية الإسلامية الحكومية ا بادنج بانجنج
}

\section{Linori Putri Bungsu}

e-mail: linoriputribungsu@yahoo.com Institut Agama Islam Negeri Batusangkar

\section{Devy Aisyah}

e-mail: devy.aisyah@iainbatusangkar.ac.id Institut Agama Islam Negeri Batusangkar

\section{Amelia}

e-mail: amelia@iainbatusangkar.ac.id Institut Agama Islam Negeri Batusangkar

ملخص: الغرض من هذا البحث هو لمعرفة تأثير استراتيجية توجيه التفكير خلال القراءة (DRTA) على فهم المقروء في المدرسـة العالية الإسلامية الحكومية ا بادنج بانجنج خصوصيا للفصل الحادي عشر. واستعملت الكاتبـة منهج البحث التجريبي. وتصيميم البحث هو تصيميم بحث تجريبي حقيقي، بنوع تصيميم مجموعاة التحكم فى مرحلة ما بعد الاختبار فقط. طريقة جمع البيانات هي الامتحان و طريقة تحليلها هي tt. وبعد أن تستعمل الكاتبـة استراتيجياة توجياه التفكير خلال القراءة (DRTA)، فيوجد معدل النتائج في الفرقة التجريبية هي .9 .9.9 ومعدل النتائج في الفرقة الضابطة هي جr، . V. ثم وجدت الكاتبة أن

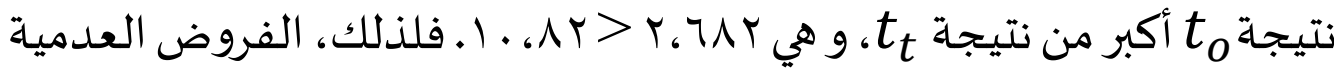
مردودة و الفروض التبادلية مقبولة. والخلاصية من هذا البحث، أن استراتيجية توجياه التفكير خلال القراءة (DRTA) تؤثر على فهم المقروء.

الكلمات الأسـاسية: استيراجية، توجياه التفكير خلال القراءة ، فهم المقروء، اللغة العربية.

Abstract: This research aims to analyze the influence of Directed Reading Thinking Activity (DRTA) strategy on reading comprehension of Arabic language at MAN I Padang Panjang. The researcher used experimental research. The design was post-test only control group of true- 
experimental design. Technique of data collection is reading test and then analyzed by using t-test. After DRTA strategy was implemented in experimental group, while control group was taught using conventional method, it was obtained that the mean score of the experimental group was 90.92 and the mean score of control class was 70.36. Then, by using t-test, it was found that tcalculated is higher than ttable, 10,82>2,682. It means that Ho is rejected and Ha is accepted. It can be concluded that DRTA strategy influences students' reading comprehension in Arabic language.

Key words: Strategy, Directed Reading Thinking Activity (DRTA), reading comprehension, Arabic language.

الكتابة. ويمكن أيضا أن تتحقق القدرة

على القراءة في شكل القراءة بصوت عال أو بصمت، والقراءة بصوت عال لا تظهر فقط فهم ما يقرأ، وقراءة بصهمت. لقد كان مفهوم القراءة بسيطا في مطلع هذا القرن اذا لم يكن يتعدى المعرد

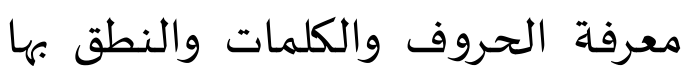
صحيحة، بمعنى أن مفهوم القراءة كان

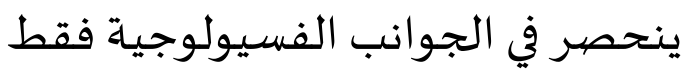
(السيد، .911: . 77). ويقصد بالقراءة فيما مضى القدرة على التعرف على الرموز المكتوبة والنطق بها. ثم تبادل هذا المفهوم، حيث أصبحت القراءة تعني

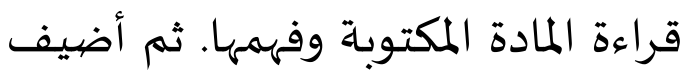
ألى ما سبق شرط اخر وهو تفاعل

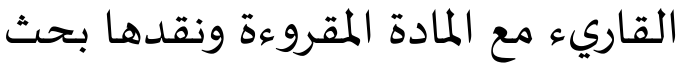

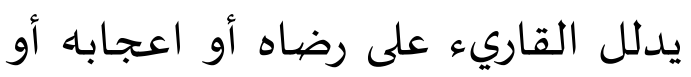
غضببه أو غير ذلك من مشيرات التفاعل حيل المادة المقروءة. (جابر، إل199 1: 1ع). وهكذا تطور مفهوم القراءة من
القراءة هي مهارة لغة واحدة

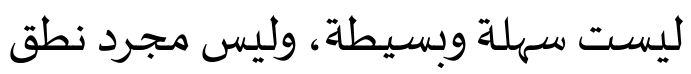

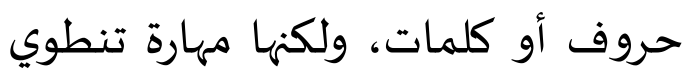
على مجموعة متنوعة من العقل والعقل. والقراءة هي نشاط يشمل جميع أشكال

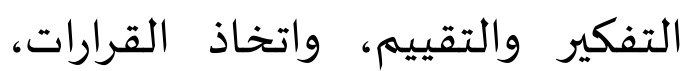
والتحليل والبحث عن حل للمشاكل. ولذالك، في بعض الأحيان، يجب على ولى لـ لهن

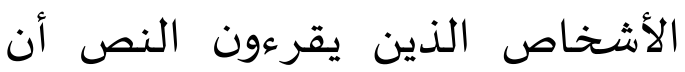

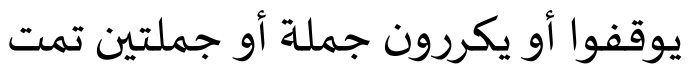

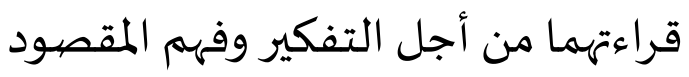

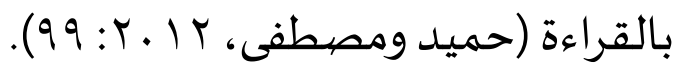

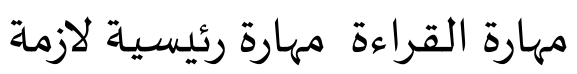
في تعليم اللغة بجانب المهارات الأخرى فهره فهي فهم المسموع والكلام والكتابة

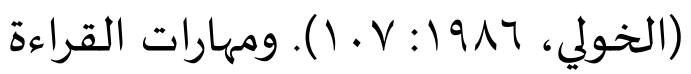
تحتوي أساسا على جانبين، وهما تحويل برنامج الكتابة إلى صوت، والتقاط معنى جميع المواقف التي يرمز إليها رمز 
التقاط الافكار الكاتب، ويعطى التقييم

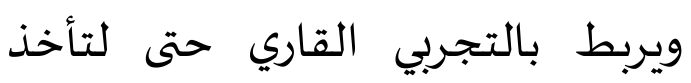
الفوائد من تلك علمية القراءة (الموجب

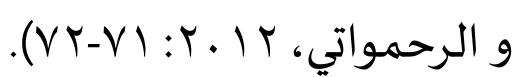
بالإضيافة إلى كون القراءة معرفة الحروف والكلمات، والنطق بها نطقا صحيحا. فهي كذلك 》 معرفة الأفكار

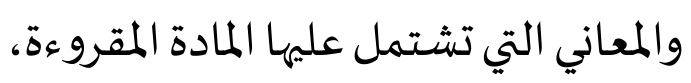
وفهمها جيدا ثم نقدها، والتمييز بين التافه منها والمفيد. بحيث قابلت الكاتبة مدرس اللغة العربية في المدرسة العالية الإسلامية الحكومية ابادانج بانجنج،

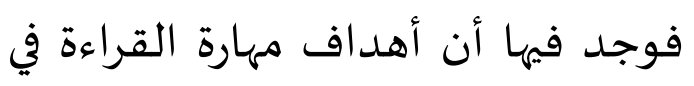

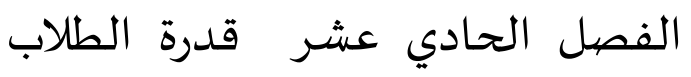
لقراءة المفردات ونصوص اللغة العربية وفهمهما.

وبناء على البيانات الواضحة وجدت الكاتبة أن كثرة الطلاب قادرون في قراءة النصوص العربية بالشكل

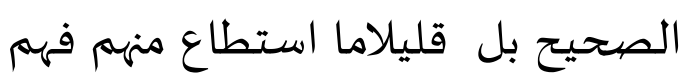
معناه. وفي أثناء التدريس يستعلئل المدرس طريقة الترجمة و والوسيلة

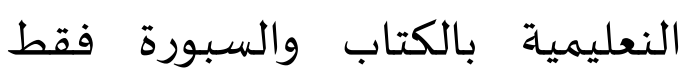
فيصبح الطلاب في هذا الفصل غير والير الحماسة ويشعر بعضهه بملال. وفي بعض الأحيان قرأ المدرس المواد، بحيث لمشيث
مجرد التعرف والنطق في العقد الأول من القرن العشرين، إلى فهم المادة المقرؤة ونقدها في العقد الثاني، ثم إلى اعتبارها نشاطا فكريا متكاملا يشير إلى حل المشكلات في العقد الثالث، وأخيرا الاستمتاع بالمقروء وتذوقة. فالقارئ الحقيقي يتعين عليه- أثناء عملية القراءة- أن يستنجدد بأقصى طاقات

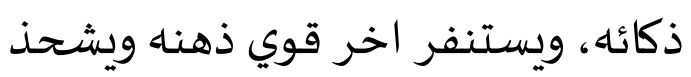

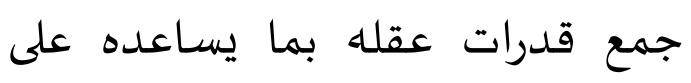
النهوض باستدعاء المعنى الذي يريده

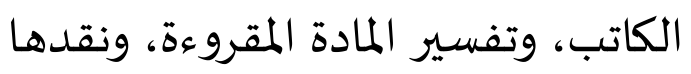
والحكم عليها، والربط بين مايقرأ وبين خبراته الخاصة. من هنا بدأ الاهتمام

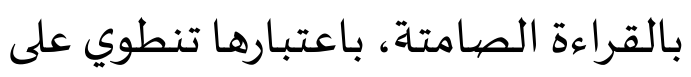

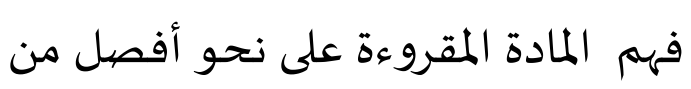

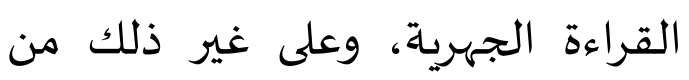

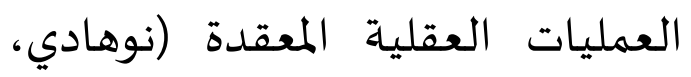
. (7T:Y. I)

القراءة في اللغة العربية تنقسم إلى قسمين: لفظ المكتوب وفهم المقروء. لفظ المكتوب هو لفظ الرمز (كتابة)

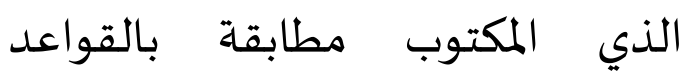
للتطبيق. وبينما فهم المقروء هو فهم شئ سلمابعه بالهواعد من قرئ. هذه بعد يشتمل عملية العقلية

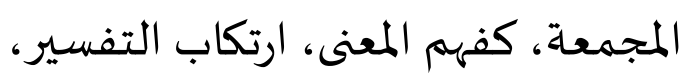


تفاصيل القراءة التي يتم قراءتها.أما أهداف فهم المقروء مما يلي: () تنمية قدرة الطلاب على القراءة وسرعتهم فيها و همون وجودة نطقة وأدائه وتمثيله للمعنى، Y ) فهمه للمقروء فيها صحيحا وتميزه بين الأفكار الأسـاسية والفرضية فياه و تكونه الأحكام النقدية عليه، و ؟r) تنمية قدرة يتيع ما يسمع وفهم فهما صحيحا ونقده

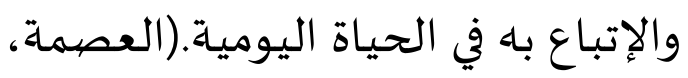

$$
\text { (1):Y. } 10
$$

ومن هذا المنطلق أرادت الكاتبة

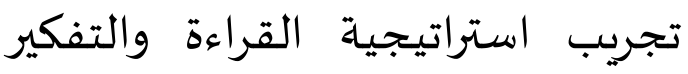
مباشرة أوتوجيه التفكير خلال القراءة ("DRTA"Directed Reading Tbinking Activity) هو يهدف على تدريب الطلاب على تركيز و التفكير بصوت عال من أجل فهم المحتوى محمل الجدمن القراءات

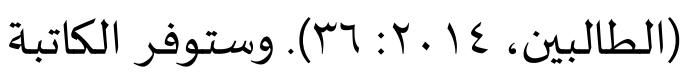
في كتاب تعليم القراءة في المدرسة الإبتدائية أن استراتيجيةتوجياء التفكير لئي خلال القراءة (DRTA)، هي استراتيجية مشاركة الطلاب في التركيز مع النص، لأن

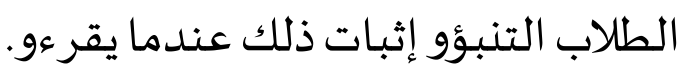

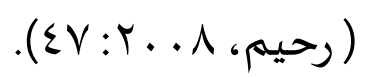

وتختار الكاتبة استراتيجية توجه القراءة نشاط التفكير (DRTA) علي فهم
يكون هناك ت تلاميذ يتحدثون مع أهدائ

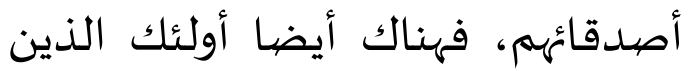
يعملون في مواضيع أخرى، وبعضهمه

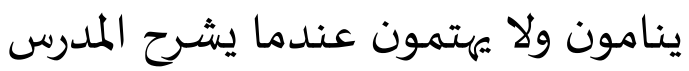

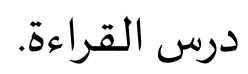

بالنسبة عن النتيجة في عملية تعليم اللغة العربية خصوصها في مهارة

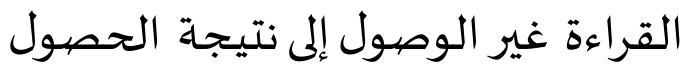

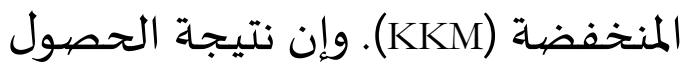
المنخفضة في هذه المادة هي V7. ونتائج

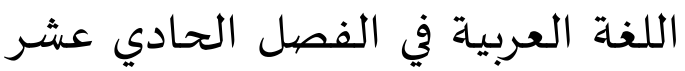
للمدرسة العالية الإسلامية الحكومية ا بادنج بانجنج خصوصا في مهارة القراءة

\begin{tabular}{|c|c|c|c|c|c|c|}
\hline الطلاب & الطلاب & غنير & ناجع & |مجتنه & فصل & رفم \\
\hline$<76$ & $>76$ & ناجي & & & & \\
\hline 10 & 15 & $40 \%$ & $60 \%$ & ro & $X \|$ IPA 1 & 1 \\
\hline 14 & 11 & $56 \%$ & $44 \%$ & to & $X \| I P A 2$ & r \\
\hline
\end{tabular}

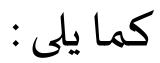

من الجدول السابق، عرف أن كثرة الطلاب راسبون في قدرة فهم مادة

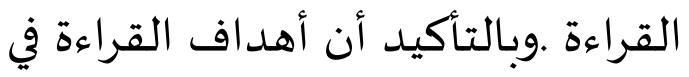
الفصل الحادي عشر للمدرسة العالية

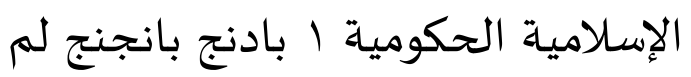

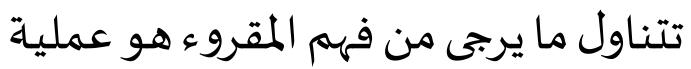

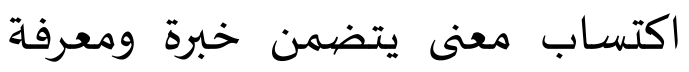
نشطة، وإتقان محتويات القراءة وفهم حبره ومعره 
الصف الحادي عشر من المدرسة العالية الإسلامية الحكومية ا بادنج بانجنج كلهم .0 طالبا. وقسمتهم الكاتبة فرقتين وهم الفصل XI. IPA ( المجموعة التجربية) بعددهم 0ب تلميذا. والفصل XI.IPA 1 To تلميذا، وتأخذهم الكاتبة عينة

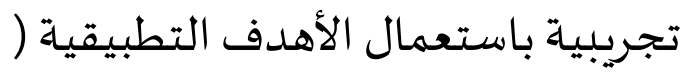
.(purposive sampling وآلة البحث في هذه المبحوثة: ( ) دليل الملاحظة: تلاحظ الكاتبة في المدرسة العالية الإسلامية الحكومية الماحفة بادنج

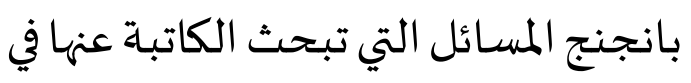
هذه الرسالة، بالي دليل المقابلة: هي تقابل

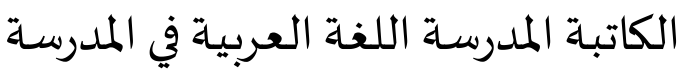
العالية الإسلامية الحكومية ا بادنج

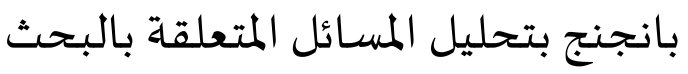
عن هذه الرسالة العلمياة، ب) أسئلة الامتحان: تقوم الكاتبة بالامتحان على الى الهاه الطلاب لمعرفة فهم المقروء في مهارة القراءة في المدرسة العالية الإسلامية الحكومية ا بادنج بانجنج. وتصحيح ألة البحث و ثقتها فيما سبق: () تأخذ الكاتبة مصدر البحث البحث للامتحان من كتاب تعليم اللغة العربية

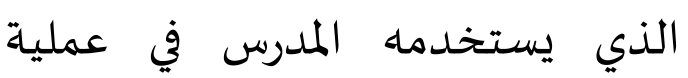

المقروء ليشـارك الطلاب للنص أو القراءة

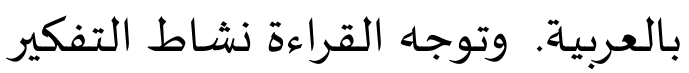
استراتيجية حديثة في تعليم (DRTA) اللغة العربية خصوصيا لفهم النصوص في المدرسة العالية. أما خطوات توجياه

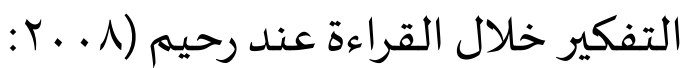

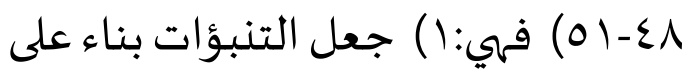

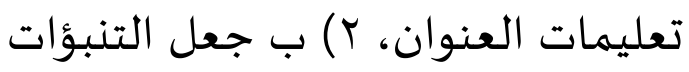

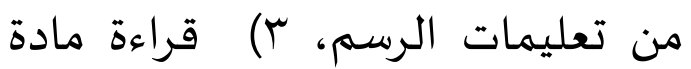
القراءة، ع) تقييم دقة التنبؤات وضبط لعبه

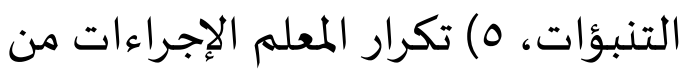
ا إلى ع، حتى جميع الدروس أعلاه تغطي. طريقة البحث بناء على المشكلات السابقة

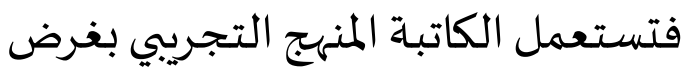
معرفة تأثير استراتيجية توجياء التفكير

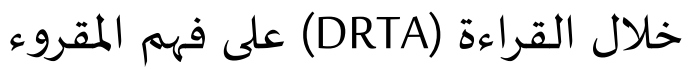

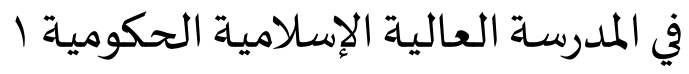
بادنج بانجنج. وفي هذه المبحوثة تقوم الكاتبة بها في المدرسة العالية الإسلامية الحكومية ابادنج بانجنج. وأما مجتمع البحث في هذه البحث فهو الطلاب في الفصل الحادي عشرفي المدرسة العالية الإسلامية الحكومية البادنج بانجج. وأما عينة البحث، فتأخذ الكاتبة الطلاب في 


\section{باسم SPSS versi 24}

وحينما استعمل الكاتبة

استراتيجية توجياء التفكير خلال القراءة في المجموعة التجريبية (DRTA) (eksperimental) بالامتحان على الطلاب. وتوجد أقصى النتائج في فهم المقروء باستراتيجية المانية توجياء التفكير خلال القراءة (DRTA) 99 وأدنى النتائج VA ومعدل النفير النتائج

$$
.9 ., 94
$$

وأما حصول الامتحان في

المجموعة الضابطة (kontrol) فوجد أن أقصى النتائج في فهم المقروء بطريقة الترجمة 10 وأدنى النتائج .7 ومعدل فئل

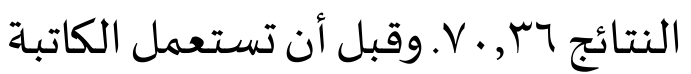

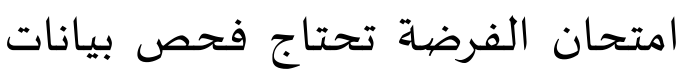
لمتغيراتXو Y. و وفي هذا الفحص تستعمل

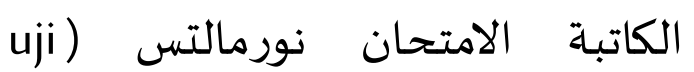
(normalitas (uji homogenitas) امتحانين هو لتأخذ الخلاصة صحيحة

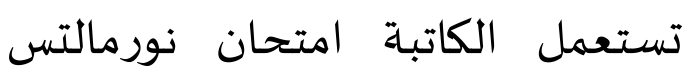

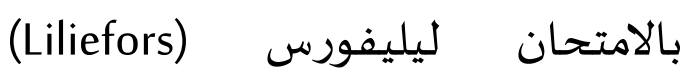
والامتحان هموجنيتس بالامتحان بارتليت (Bartlett).

قد استعملت الكاتبة امتحان
التعليم، ب) وزعت الكاتبة أسئلة الامتحان إلى المدرّس الذي يعلّم تعليم

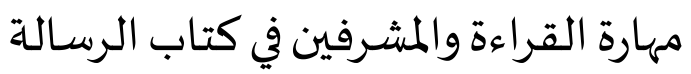

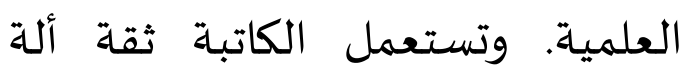
البحث بطريقة المتواز هي توجد تساوي الفهم من شخص البحث و امتحان مواد

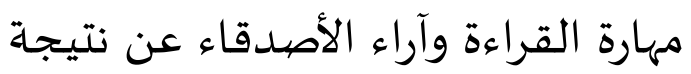
التجريبية في عينة. وفي هذه البحث تستعمل الكاتبة تصميم البحث هو تصيميم بحث تجريبي حقيقي (true) eksperimental design

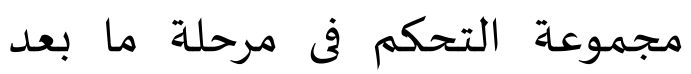

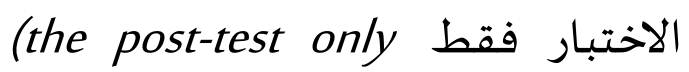
.control group design)

و لتحليل النتيجتين استعملت الكاتبة الرمز لمعرفة تأثير استراتيجية توجياه التفكير خلال القراءة (DRTA) على فهم المقروء في المدرسة العالية الإسلامية الحكومية ا بادنج بانجنج. وجعلتها أسـاسية في استنباط الخلاصية الحكاه uji ) من البحث. الامتحان نورمالتسانس uji ) و امتحان هموجنيتس (normalitas غوomogenitas غير هموجنيتس من الاستفتاء تستعمل

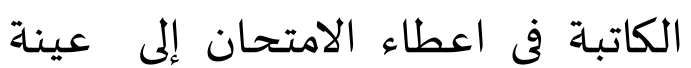
البحث، فتستعمل الكاتبة بالكومبيوتر 
.096Test Statistic $=.200^{c, d} g .071$ .Asymp. Sig. (2-tailed)= نتيجة البحث ومناقشته بناء على أسئلة البحث في منهج البحث تجرّب الكاتبة في تعليم فهم المقروء في المجموعة الضيابطة باستعمال طريقة الترجمة، فوجدت الكاتبة نتائج فهم المقروء، بأقصى النتائج 85 وأدناها

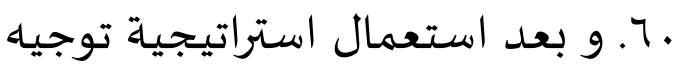
التفكير خلال القراءة (DRTA) في تعليم مهارة القراءة (فهم المقروء) في المجموعة التجريبية فوجدت فيها أقصى النتائج 99 و أدناها 78. ففسرت الكاتبة نتيجة

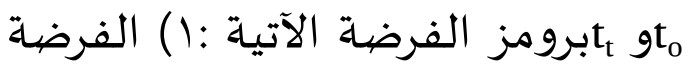
العدمية (Ho) مردودة إذا كانت النتيجة

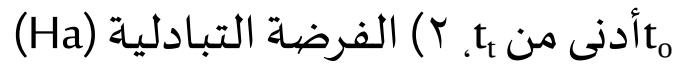
مقبولة إذاكانت النتيجة من أكبر من أt بعد ما حللت الكاتبة هذه البيانات بالرمز test فحصلت الكاتبا نتيجة 10,82 to الذين (degree of freedom) df يتعلمون بالرمز df = n تفسر الكاتبة هذه النتائج في df25 25-2+ فلذلك df =d8 وفي جدول to الدرجة ه \% هي \% 2,0111 وفي الدرجة
نورمالتس البيانات بكيفية امتحان ليلفور (Liliefors) لمعرفة نورمالأو غير بوني نورمال من الامتحان تستعمل الكاتبة في إعطائه إلى عينة البحث، فتحللت الكاتبة بالحاسوب باسم SPSS24

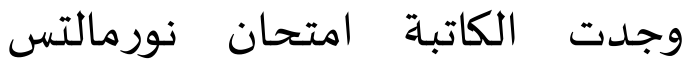
للمجمموعة التجريبية , و، وSD=6.33061و،90.9200Mean= ،Most Ekstreme Absolut=. 168 $=---168$ وDifferences Positif $=.105$ .168Test وDifferences Negatif .Asymp. Sig. (2- . =.068c ،Statistic $=$ هكذا حاصل الامتحان (ailed) نورمالتس في متغير X (نتائج فهم المقروء باستراتيجية توجياء التفكير خلال القراءة (DRTA)). وما أصبح ذلك، لمعرفة نورمال أو

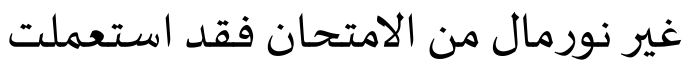
الكاتبة في إعطاء الامتحان إلى عينة البحث، فحللت الكاتبة بالحاسوب باسم SPSS24 فوجدت الكاتبة امتحان نورمالتس للمجموعة الضابطة هي:Mean=70.3600، نورماسن MostEkstreme g، $=$ Differences Positif gAbsolut $=.096$ DifferencesNegatif $=-\quad$ g، $=.096$ 
المدرسة العالية الإسلامية الحكومية | بادنج بانجنج. واستفادة هذا البحث أن الن الندانيه

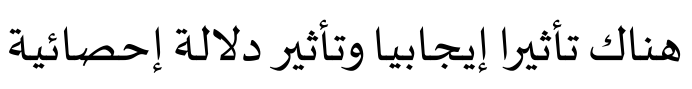
بين المجموعة التجربية (يعنى الطلاب الذين يتعلمون باستعمال استراتيجية توجيه التفكير خلال القراءة (DRTA) والمجموعة الضيابطة (الطلاب الذين يتعلمون باستعمال طريقة الترجمة) في

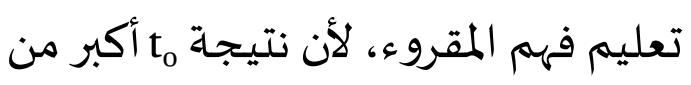

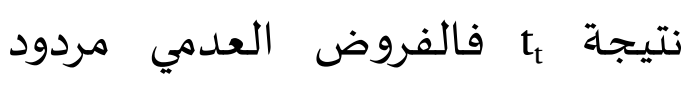
والفروض التبادلي مقبول.

الخلاصية

بناء على نتائج البحث السابقةة، فوجدت الخلاصاة ، كما تلي: 1. يوجد فرق في فهم المقروء بين

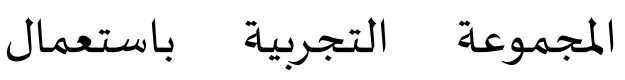

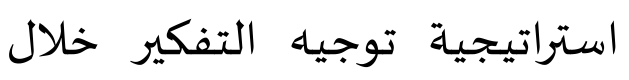

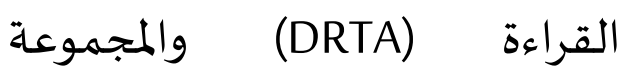
الضيابطة باستعمل طريقة الترجمة في المدرسة العالية الإسلامية الحكومية ا بادنج بانجنج. r. يوجد فرق ذات دلالة إحصائية في فهم المقروء بين المجموعة التجريبية باستعمال استراتيجية توجياه التفكير خلال القراءة (DRTA)
1\% هي 2,682 تعد ما قارنت الكاتبة

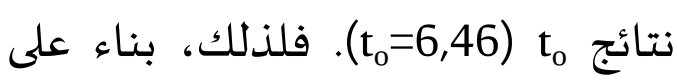
البيانات السابقة فعرفت الكاتبة أن

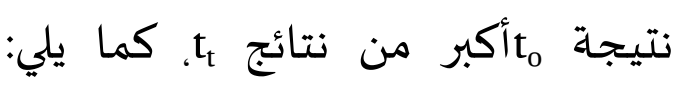
.NT.1. > TNT. T

ومن حصول انتهاء الرمز السابق

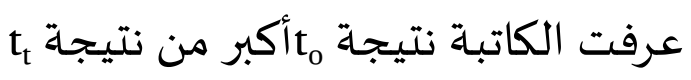
فالفرضة العدمية Ho مردود والفرضية التبادلية Ha مقبول، بعدما نظرت الكاتبة نتيجة امتحان الفرضية السابقة

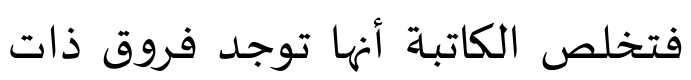
دلالة إحصائية لدى الطلاب في فهم المقروء بين استعمال استراتيجية توجياه

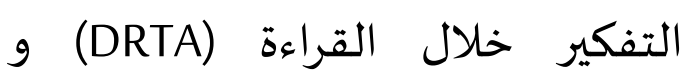
باستعمال طريقة الترجمة في المدرسة التركة

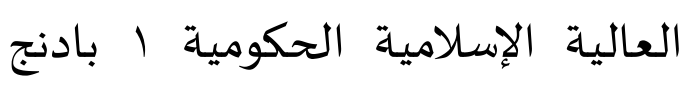
بانجنج. ومن تحليل البحث اتضح أن هناك فرق بين نتائج الطلاب الذين يتعلمون

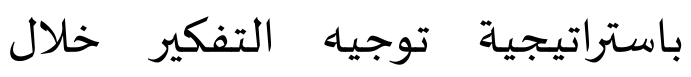
القراءة (DRTA) والطلاب الذين النين يتعلمون باستعمال طريقة الترجمة. وكذلك أن استعمال استراتيجية توجيه التفكير خلال القراءة (DRTA) تؤثر تأثيرا إحصائيا على نتائج الطلاب في فهر المهر

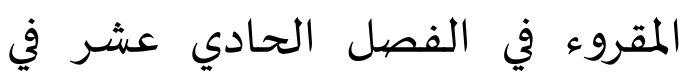
8 |Linori Putri Bungsu, Devy Aisyah, Amelia 
لدي تلاميذ مدرسة محمدية

المتوسطة لشيفوتات. رسالة

بكالوريوس .جامعاة شريف هداية

الله الإسلامية الحكومية جاكرتا.

Mujib, F. dan Rahmawati, N. 2012.

Permainan Edukatif Pendukung

Pembelajaran Bahasa Arab (2).

Yogyakarta: DIVA Press.

Mustofa, B. \& Hamid, A. 2016. Metode dan Strategi Pembelajaran Babasa Arab. Malang: UIN-Maliki Press.

Mustofa, S. 2011. Strategi Pembelajaran Bahasa Arab Inovatif. Malang: UINMaliki Press.

Rahim, F. 2008. Pengajaran Membaca di Sekolah Dasar. Jakarta: Bumi Aksara.

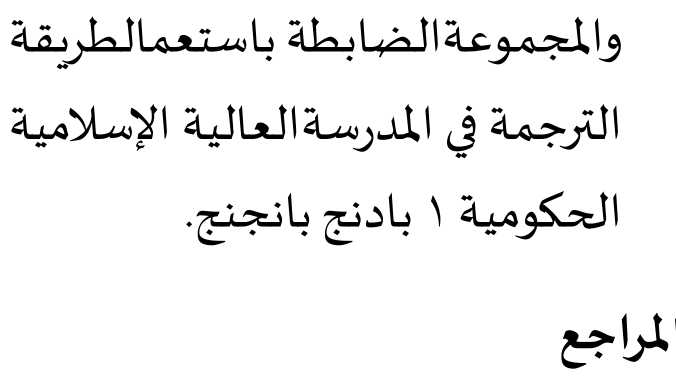

الخولي، ع. 71919 أساليب تدريس اللغة

العببية. الرياض: المملكة العربية

السعودياة.

السيد، أ. . 191 ا. طرائق تدريس اللغة

العربية وادابها. بيروت: دار العودة.

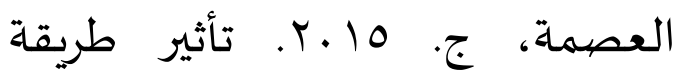

تحليلية على قدرة الطلاب في فهم

المقروء " بحث تجربي" في مدرسة

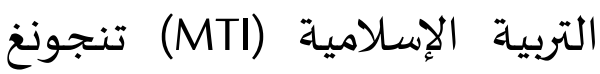

برولاك. رسالة بكالوريوس. جامعة المساية

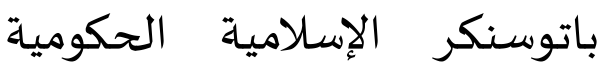

باتوسنكر.

جابر، و. 1991 ـ أساليب تدرليس اللغة العبية. الأردن: عمان.

نورهادي. II ــ ا. الموجه تعليم المهارات

اللغوية لغير الناطقين بها. مالانق:

مطبعة جامعة مولانا مالك إبراهم

الإسلامية الحكومية.

زكية ،ف. 19 إ. فعالية استخدام

استراتيجية توجياه التفكير خلال

القراءة (DRTA) في مهارة القراءة 